Fecha de recepción: diciembre 2017 Fecha de aceptación: marzo 2018 Versión final: julio 2019

\section{El rol de los intermediarios culturales en la producción de la "creencia colectiva": la conformación de un circuito de diseño en Quilmes}

Gabriela C. Alatsis *

Resumen: Este artículo propone examinar la especificidad de los bienes simbólicos, su relación con el mercado y la construcción de la creencia colectiva en el valor de los productores y de los objetos que éstos fabrican, en base a los aportes de Pierre Bourdieu a la sociología de la cultura. Para abordar dichas cuestiones analizaré, puntualmente, el rol que desempeñaron los intermediarios culturales, en el marco de diversas políticas públicas culturales, para lograr constituir y legitimar un circuito de diseño de indumentaria y accesorios en el partido de Quilmes, ubicado en la zona sur del conurbano bonaerense, entre 2008-2015.

Palabras clave: bienes simbólicos - creencia colectiva - intermediarios culturales - diseño - valor - circuito - políticas públicas.

[Resúmenes en inglés y portugués en la página 192]

${ }^{(*)}$ Gabriela Alatsis es Licenciada en Sociología por la Universidad de Buenos Aires y cursa el Doctorado en Ciencias Sociales en esa casa de estudios. Es becaria doctoral del CONICET y docente regular de la materia Introducción a la Sociología en la Universidad Nacional Arturo Jauretche. Correo: gcalatsis@gmail.com

\title{
Introducción
}

A principios de los años 2000, la categoría "diseño" adquirió visibilidad y relevancia en la escena pública, en particular en la Ciudad Autónoma de Buenos Aires (CABA). Junto a este proceso, surgieron nuevos espacios de circulación de productos con un alto valor simbólico, denominados socialmente de "diseño", y distintas formas de valorización de los mismos. Desde la prensa especializada, por ejemplo, se creó la categoría "diseño de autor" para referirse a esta producción con atributos específicos.

Con el desarrollo de estos fenómenos emergieron asimismo una multiplicidad de trabajos que se dedicaron a su estudio. Algunos trabajos, centrados en el ámbito y quehacer profesionales, analizan, desde una mirada sociológica, la conformación y consolidación del campo del diseño de indumentaria y su valoración desde diversos sectores (Miguel, 
2009, 2011, 2013), o bien las prácticas de jóvenes diseñadores industriales y sus estrategias de inserción en el mercado, a partir de iniciativas autogestionadas (Correa, 2018). Otros estudios, en cambio, parten de una perspectiva etnográfica y examinan, por un lado, la producción, circulación y consumo de objetos e indumentaria de diseño -y los repertorios morales y formas de distinción que intervienen en esos procesos (Vargas, 2013)-y, por el otro lado, los sentidos sociales que se otorgan a las mercancías clasificadas "diseño" y los modos en que el espacio social en el que se comercializan influye en la configuración de dichos sentidos (Guerschman, 2010). Salvando sus diferencias, todos los estudios convergen en señalar el crecimiento que han tenido las distintas ramas profesionales del diseño en CABA, a inicios de la década del 2000, y asimismo destacan que junto con la clasificación de ciertos bienes como de "diseño", surgieron espacios propios de venta de este tipo de productos, localizados en el barrio porteño de Palermo, particularmente en un área denominada "Palermo Viejo". En relación a la incidencia de políticas públicas en el desarrollo de los emprendimientos productivos dedicados al diseño, en su mayoría indican que ésta fue escasa o incipiente.

Sin embargo, a partir de mediados de la década, estas políticas adquirirían un papel más importante y se propagarían hacia otras zonas del país. En relación a estas transformaciones, propongo en este artículo analizar, desde una perspectiva bourdieuseana, la conformación de un circuito (Zelizer, 2008) de diseño -de indumentaria y accesorios-, localizado en el partido de Quilmes, en la zona sur del conurbano ${ }^{1}$. Este circuito estuvo acompañado por una política pública de fomento al diseño, que tuvo lugar entre 2008 y 2015, lo que me llevará también a examinar las estrategias implementadas por las intermediarias culturales -coordinadoras del programa- para conformarlo y lograr su legitimidad. En este sentido, algunas nociones centrales de la obra del sociólogo Pierre Bourdieu (1990a, 1990b, 1995, 2012, 2014a, 2014b) -como la creencia colectiva en el valor del producto y de los productores; la especificidad de los bienes simbólicos; y la denegación de la "economía”- serán sumamente operativas para analizar las cuestiones propuestas. En cuanto a la metodología, me valdré de distintas herramientas metodológicas cualitativas -entrevistas en profundidad a las emprendedoras que integraron ese circuito, como así también a las organizadoras del mismo; charlas informales con residentes de los barrios de Quilmes, donde se llevaron a cabo los principales eventos de diseño; y relevamiento de diversos documentos, por ejemplo: informes de gestión de la política cultural y catálogos de difusión de los emprendimientos, que formaron parte del circuito-.

\section{La expansión del diseño: el protagonismo de las políticas públicas}

A mediados de la década del 2000, y especialmente a partir de la primera presidencia de Cristina Fernández de Kirchner, la cultura adquirió un papel central en la agenda pública y se promovieron un conjunto de políticas públicas de fomento a las industrias culturales y, en particular, al diseño ${ }^{2}$. En este marco, el presupuesto nacional destinado al área de cultura se incrementó año tras año y se crearon diversas instituciones dedicadas al desarrollo cultural, como el Ministerio de Cultura de la Nación y el Mercado de Industrias Culturales Argentinas (MICA), que tuvo desde sus inicios, según documentos oficiales y entrevistas a 
funcionarios, el objetivo de "mejorar la producción, visibilidad y comercialización de los productos de las industrias culturales en cada región del país"3.

Por otra parte, en relación al contexto económico, hacia fines del 2008, la industria nacional sufrió una desaceleración en su crecimiento, debido, entre otros factores, a las repercusiones que tuvo la crisis internacional en el país y al conflicto del gobierno con los sectores agropecuarios, hechos que provocaron inestabilidad política y social (Kulfas, 2017; CENDA, 2010). La industria textil y de la indumentaria -industrias trabajo-intensivas, encargadas mayormente de abastecer al mercado interno- fueron unas de las más afectadas (Castells y Schorr, 2015; Kulfas, 2017). Para contrarrestar la pérdida de actividad en el sector y recuperar el crecimiento, objetivo que se alcanzaría en 2010, el gobierno implementó una batería de "políticas expansivas diversificadas" (Aronskind, 2012), dirigidas a estimular la demanda interna y a regular el comercio exterior. Fue así que se impusieron ciertas restricciones a las importaciones, por medio de las licencias no automáticas a la importación, para disminuir los efectos negativos sobre la producción y el empleo. Si bien el régimen de licencias no automáticas existe desde 1999, recién a partir de 2009 adquirió especial relevancia como política para proteger a la producción interna en el contexto de crisis (Amar y Tumini, 2012: 171).

En suma, el circuito de diseño en Quilmes pudo constituirse, como se verá más adelante, gracias a las estrategias de valorización simbólica impulsadas por las intermediarias culturales, pero así también debido a un contexto particular de "jerarquización" de las industrias culturales - a través de la creación de políticas públicas- y de promoción de medidas de corte "proteccionistas" que limitaron el ingreso de productos manufacturados, como prendas de vestir y accesorios. Al igual que plantean los estudios sobre el diseño a principios de los años 2000, anteriormente citados, la disminución de las importaciones dio lugar al crecimiento de la producción nacional de objetos e indumentaria con el atributo de "diseño" (Miguel, 2013; Vargas, 2013). Pero, sin embargo, para el caso del circuito de diseño quilmeño, las políticas públicas -en particular el programa DAS (Diseños al Sur), al que me referiré en el próximo apartado- adquirieron un papel central en la expansión del diseño hacia otras zonas del país, distintas a CABA.

\section{El Programa DAS (Diseños al Sur)}

En ese marco de “jerarquización” cultural, la Subsecretaría de Cultura y Educación del Municipio de Quilmes adquirió, en el 2008, el estatuto de secretaría. Desde allí se creó, en ese mismo año, por la iniciativa de dos funcionarias -ambas provenientes del ámbito del diseño- el Programa DAS (Diseños al Sur), que funcionó hasta 2015. Este programa, dirigido a jóvenes emprendedores y/o diseñadores profesionales, se orientó hacia el fomento de la producción y circulación de productos de "diseño", correspondientes a los rubros de indumentaria, textil, calzado, accesorios y objetos decorativos. Gracias a la obtención de mayor presupuesto y a la mayor demanda de participación de los productores, el programa se convertiría, en 2011, en un área independiente dentro de la secretaría, denominándose: Dirección Operativa de Industrias Culturales - DAS. 
Según los catálogos del programa (2011, 2012, 2013), el DAS se propuso "hacer de Quilmes una ciudad referente en materia de diseño". Al respecto, una de las coordinadoras plantea:

Desde un primer momento el objetivo era hacer una especie de, si querés, algo similar o con un poco de miras a hacer algo como era el Centro Metropolitano de Diseño, en la zona. Y surge porque notábamos que no había nada, digamos, que abastezca a toda la gente relacionada con el diseño en cuanto a capacitaciones, no solamente el tema ferias.

Siguiendo esta línea, la otra coordinadora también señala:

Se nos ocurrió gestionar algo desde el Estado y con base territorial, local, porque venía experimentado desde lo privado que quizás había una mirada muy fuerte hacia lo que es CABA, y tratar de todo lo que se producía en el conurbano insertarlo en la Ciudad de Buenos Aires. Entonces investigando por ahí, con estas ferias privadas que yo hacía en La Plata, me daba cuenta de que había mucha producción y que veníamos post crisis 2001 y el boom del diseño y toda esta movida que se generó. Entonces había mucha producción en ese sentido, pero todo con la mirada puesta en la Ciudad de Buenos Aires. Entonces la propuesta, al principio, siendo parte del Municipio, fue mostremos acá qué hay, hagamos una sede de conurbano sur, donde se pueda empezar a mostrar que toda esta producción que se muestra divina y fabulosa en la Ciudad de Buenos Aires es de acá.

Sin embargo, las coordinadoras del DAS observaron ciertas "limitaciones", en los primeros ensayos de implementación del programa, para poder cumplir con estas expectativas. Según destaca una de ellas:

Fue un proceso largo, porque el público de la Ciudad de Buenos Aires no es el público del conurbano y entonces fue todo un aprendizaje para lo que era el comprador local...que sabía que si se lo encontraba [al diseño], se lo encontraba allá. Si era de acá, era como "trucho".

Frente a estos "obstáculos", que señalaron las funcionarias, en los inicios del trabajo de campo, las preguntas de la investigación se dirigieron a indagar acerca de las estrategias que se propusieron para instalar "un polo de diseño en Quilmes", que diera visibilidad a los productores locales, pero que también pudiera estimular a un público que, al parecer, no estaba preparado para consumir ese tipo de productos en su lugar de residencia. En este sentido, el rol de las coordinadoras -en tanto intermediarias culturales- fue central para articular la producción con el consumo y consolidar un circuito de diseño en la zona. Como señala Bourdieu en La distinción (2012), los intermediarios culturales encarnan un segmento de la "nueva pequeña burguesía" que se "realiza en las profesiones de presentación y representación (...) y en todas las instituciones dedicadas a la venta de bienes y 
servicios simbólicos (...)" (Bourdieu, 2012: 420). Estos agentes son el eslabón que conecta a los productores con los consumidores (Negus, 2002). Partiendo de su expertise en el ámbito cultural, los intermediarios culturales le atribuyen a los bienes y servicios determinados sentidos y valores, que luego son exhibidos a los posibles compradores (Du Gay et al., 1997). No obstante, las estrategias puestas en práctica por los intermediarios culturales, para promover a los productores y a los bienes que éstos fabrican, no se explican simplemente por sus destrezas personales sino que se relacionan con el lugar que ocupan en el campo cultural y las lógicas de funcionamiento del mismo. Pero, antes de profundizar en estas cuestiones, examinaré en el siguiente apartado las características de los bienes simbólicos y su especial vínculo con lo económico.

\section{Los bienes simbólicos: "realidades de doble faz"}

Como señalan diversos estudios de Bourdieu sobre el campo artístico y cultural, los bienes simbólicos son "realidades de doble faceta", mercancías y significaciones, cuyos valores simbólico y comercial permanecen relativamente independientes (Bourdieu, 1995, 2014a, 2014b). Según esta perspectiva, se generan dos modos de producción y circulación que obedecen a lógicas inversas. Una que se guía por los valores del desinterés y rechaza la "economía" (lo comercial) y el beneficio "económico" (a corto plazo), al mismo tiempo que orienta la producción hacia la acumulación de capital simbólico y genera su propia demanda a largo plazo. Las empresas, que responden a esta lógica, son empresas de "ciclo de producción largo", ya que ellas mismas construyen la demanda de sus productos y los beneficios "económicos" se alcanzan solamente a largo plazo y por la acumulación de capital simbólico. En cambio, la lógica opuesta es la "económica", que convierte el comercio de bienes culturales en un comercio como cualquier otro, otorgando prioridad al éxito inmediato y temporal. Las empresas cercanas a esta lógica son, según Bourdieu, "de ciclo de producción corto": se ajustan a una demanda prexistente y cuentan con espacios de comercialización y difusión, con el propósito de garantizar el reintegro acelerado de los beneficios "económicos". Bourdieu asocia estas dos lógicas con dos campos diferentes: "el campo de producción restringida" -sistema que produce bienes simbólicos (e instrumentos para su apropiación) destinados a un público de productores de ese tipo de bienes- $y$ "el campo de la gran producción simbólica" -con vistas a la producción de bienes simbólicos destinados al "gran público"-. En este sentido, una de las coordinadoras afirma que la intención del DAS fue:

Instalar en la zona esta cuestión de aceptar, aprender sobre el diseño, qué otras posibilidades había además de lo industrializado, comprado en Once. También había que mostrarle a la gente, bueno, miren estas opciones de indumentaria que hay a las marcas grosas. ¿Valen? ¡Sí, valen! Son caras, no valen 2 mangos, porque es un diseño...

Por ende, había un claro afán, de parte de las gestoras del DAS, de diferenciar la producción que el programa promovía tanto de bienes que se comercializaban de forma masiva 
en los negocios del barrio porteño de Once, como de los de las marcas ya establecidas en el mercado que circulaban en los shoppings. Los productos de "diseño" se relacionaban entonces con lo "diferente" y "único", y se oponían a lo comercial y masivo (Guerschman, 2010; Correa, 2018; Miguel, 2013; Vargas, 2013). Al respecto, una de las emprendedoras que participaron del DAS afirma:

Mucha gente no valora el valor agregado del diseño. Ven tus productos, que salen más caros, y dicen: “en Once lo consigo más barato". Pero yo ahí les explico que en Once hacen 100 baberos iguales y además en una fábrica de muchas personas. Yo, en cambio, hago piezas únicas y además corto todo y hago todo yo sola.

En la presentación del primer catálogo del programa, que se abocó especialmente a exponer sus objetivos, se enfatiza la importancia de dar a conocer el diseño local, porque esto implicaría también dar a conocer la identidad de los productores. Sin embargo, también esta política pública esperaba "generar un trato directo entre diseñadores y productores locales y los diferentes sectores comerciales zonales (...) interesados en tomar contacto con esta rama productiva en expansión". Esto indica que no sólo el DAS tenía como meta aglutinar a un determinado tipo de producción que se orientaba a la búsqueda de "las distinciones culturalmente pertinentes" (Bourdieu, 2014a: 93), que en este caso estarían relacionadas con la elaboración de bienes que incorporaran el atributo del "diseño", sino que tuvieran asimismo un valor "económico" y que fueran susceptibles de despertar el interés de un segmento específico del mercado.

Pero, ¿cómo se puede lograr la confluencia de estas dos dimensiones -la "económica" y la cultural- que, como ya mencioné, mantienen una relativa independencia entre sí? Diversos trabajos utilizan conceptos como "el recurso de la cultura" (Yúdice, 2008) o la "culturalización de la economía” (Du Gay, 2003), para aludir a la creciente importancia que adquirió la cultura en los ámbitos político y económico en la época contemporánea. No obstante, según el enfoque de Bourdieu, para que la imbricación entre estas "dos facetas" de los bienes simbólicos resulte "efectiva" deben darse determinadas condiciones. "El capital 'económico' sólo puede proporcionar beneficios específicos ofrecidos por el campo -y al mismo tiempo los beneficios 'económicos' que a menudo éstos reportarán a largo plazo- si se reconvierte en capital simbólico" (Bourdieu, 1995: 224). Entonces la única acumulación legítima en el campo cultural -tanto para los productores como para los intermediarios culturales- es "hacerse un nombre", un capital de consagración que permita consagrar objetos o personas, es decir, otorgar valor y obtener, luego, los beneficios de esta operación (Bourdieu, 1995, 2014a). Así, este "comercio de objetos de los que no hay comercio" tiene éxito, simbólico e incluso "económico", sólo si está orientado por el dominio práctico de las leyes de funcionamiento del campo de producción y circulación de los bienes culturales. Es decir, "por una combinación (...) del realismo que implica concesiones mínimas a las necesidades "económicas" denegadas y no negadas y de la convicción que las excluye” (Bourdieu, 2014a: 155). 


\section{La creencia colectiva en los creadores}

Como señalé previamente, la posesión del capital simbólico es lo que permite, en el campo cultural, otorgar valor a los productos y productores. Pero, ¿de qué modo se genera esa consagración y quiénes intervienen en ella?

En diversos trabajos, Bourdieu (1990a, 1990b, 1995, 2014b) se pregunta "¿quién creo al 'creador'?" y se opone, al igual que lo hizo Roland Barthes (1968) en "La muerte del autor", a la noción decimonónica del "genio creador", increado 4 . En lugar de considerar al autor como único responsable de la producción del valor de su obra, Bourdieu sostiene que son múltiples los agentes que se ven envueltos en este proceso. Marchands, editores, intermediarios culturales consagran los productos al introducirlos en el mercado mediante la publicación, exposición o puesta en escena. Sin embargo, estos actores no sólo procuran un valor comercial a la obra, sino que proclaman el valor del autor que defienden, ofreciendo como garantía de dicha "apuesta" el capital simbólico que han acumulado, es decir, su "prestigio" (Bourdieu, 2014b). Por ende, lo que crea el valor de la obra no es su rareza o unicidad, sino la rareza del productor, manifestada en la firma.

Ahora bien, volviendo a las preguntas que dieron inicio a este apartado, ¿de qué modo los intermediarios culturales logran su reconocido poder de consagración? Según plantea Bourdieu, la "autoridad" del que autoriza, del que "descubre" a los productores, "es un valor fiduciario que sólo existe en virtud de la relación con el campo de producción en su conjunto" (Bourdieu, 2014b: 158). La homología que el autor establece, entre los actos considerados "mágicos" y los actos de consagración que se producen en el campo cultural, es útil para comprender el componente colectivo que existe en la creación del valor de los productos y productores y, asimismo, en la creencia en dicho valor. Partiendo de las reflexiones de Mauss (1979:50) -quien destaca que "[1] os actos de cuya eficacia no cree todo un grupo no son mágicos"-, Bourdieu afirma que "[e]l principio de eficacia de todos los actos de consagración no es otro que el campo mismo (...)” (Bourdieu, 2014b: 159). Por su parte, la antropóloga Laura Colabella (2014) plantea, al analizar un acto de brujería, que más que interpretar el discurso, lo central es entender cuál es la posición social de cada uno de los participantes en ese contexto y situación concreta. De esto se deduce que no se trata de conocer los atributos específicos del mago, del brujo o de los intermediarios culturales, sino de examinar los fundamentos de la creencia colectiva o, como indica Bourdieu, del "desconocimiento colectivo", colectivamente producido y mantenido, que es la base del poder del que estos agentes se apropian. En efecto, si resulta imposible comprender el acto de consagración de los bienes y productores sin considerar a los agentes que intervienen en él, es porque el poder de estos agentes es una "impostura legítima", colectivamente desconocida y, por ende, reconocida (Bourdieu, 1995: 256). El trabajo de los intermediarios, en términos de Negus (2002), implicaría entonces el "ocultamiento" del conocimiento que estos actores poseen para lograr la articulación entre producción y consumo.

En conclusión, para analizar el valor de los bienes culturales, desde esta perspectiva, sería preciso considerar el sistema de relaciones objetivas que constituyen al campo cultural, las luchas que en él se producen y la forma específica de creencia que en él se engendra. Lo dicho implicaría entonces dejar de lado una lectura que privilegiara, como explicación de 
ese valor, las características materiales de los objetos y/o los atributos "únicos e irrepetibles" del productor.

\section{Estrategias de valorización del diseño quilmeño}

Siguiendo los planteos de Bourdieu, mencionados anteriormente, examinaré en este apartado las distintas estrategias, puestas en práctica por las coordinadoras del DAS, para atribuirle valor a los productos de "diseño" y a los emprendedores locales y, así, posicionar a Quilmes como un "polo de diseño" en el conurbano sur. Las iniciativas se enfocaron también en atraer al público quilmeño para que estuviera dispuesto a acceder a estos nuevos consumos.

El corpus de análisis tomado para esta investigación está compuesto por unidades productivas dedicadas a la confección de indumentaria y accesorios. En su mayoría, las emprendedoras a cargo de dichas unidades son mujeres, de entre 30 y 40 años de edad. Provienen de familias de comerciantes y aprendieron el oficio de la costura o del tejido en sus hogares. Sus trayectorias educativas son dispares, algunas son diseñadoras de indumentaria egresadas de la Universidad de Buenos Aires, mientras que muchas otras estudiaron en la Escuela Municipal de Bellas Artes de Quilmes, cursaron carreras terciarias de diseño de indumentaria, o tomaron distintos cursos de diseño. El Programa DAS cumplió sus expectativas de generar un circuito dedicado al diseño -que logró año a año aumentar la asistencia tanto de productores como de consumidores-, en el que los productores tuvieron una participación activa y además se sintieron pertenecientes a ese ámbito. No obstante, la mayor parte de las productoras se identificó, en las entrevistas, con la categoría de "emprendedora", que refiere a impulsar un proyecto creativo propio y encargarse de la gestión total del producto (Miguel, 2013), y, en menor medida, con el mote de "diseñadora", asociado más a la carrera profesional. Lo que indica que el empeño de las intermediarias culturales por denominar a las productoras como "diseñadoras del DAS" es un elemento más dentro de la trama de valorización simbólica que ellas construyeron, y que se verá a continuación.

\section{De Palermo a Quilmes: ferias, selección y jerarquización del territorio}

Una de las primeras acciones, que contribuyó a la puesta en valor de los productos, fue invitar a diseñadores que tenían locales, de sus propias marcas, ubicados en la zona de "Palermo Viejo", en el barrio porteño de Palermo. Como señala una de las coordinadoras, "íbamos a Palermo a un negocio, mirábamos en uno de esos negocios de diseño cosas que estaban buenas de tal rubro. 'Listo, buenísimo'. Y los invitábamos."

Otra de las formas, de "dar a conocer el diseño local", fue organizar ferias de exhibición de la producción de los distintos emprendimientos que las coordinadoras convocaban. Las ferias de diseño, como señalan los catálogos, eran "un ámbito en donde se concentran la demanda y la oferta en un lugar común". Además, suponían un "paseo de diseño donde el usuario concurre con el propósito de conocer lo último en tendencias y los expositores tienen la posibilidad de mostrar su producto y generar nuevos contactos". 
Sin embargo, no sólo los nuevos contactos revalorizaban la posición de los productores, sino que, según las coordinadoras, estar en las ferias les permitía "defender y explicar" sus productos -el concepto de la marca, los materiales, el precio-.

Asimismo, las intermediarias culturales plantean que compartir la situación de feria les abría a los emprendedores la oportunidad de conocer información útil sobre temas relativos a la producción y venta -espacios para comercializar los productos, lugares de venta de telas más baratas, capacitaciones de diseño-, que era difundida por las coordinadoras o también por los propios emprendedores. En este sentido, como indica la antropóloga Susana Narotzky (2016), las actividades económicas, de pequeña escala, en áreas de producción descentralizada, suelen están atravesadas por fuerzas "extra-económicas" (de carácter moral, emocional y social). Por ende, los emprendedores a la hora de fabricar y comercializar sus productos, no sólo se veían envueltos, en términos de Bourdieu, en un vaivén entre el interés "económico" y el desinterés, opuesto a todo beneficio "económico" inmediato, propio de la lógica del campo cultural, sino también en una oscilación entre el interés y el afecto o la "reciprocidad", que los inducía, por ejemplo, a compartir información, en términos de una emprendedora, "jugosa", con sus pares (Narotzky, 2016).

Las ferias se hacían normalmente en la Casa de la Cultura, ubicada en el centro de Quilmes, y para participar de ellas los emprendedores debían pasar por una instancia de selección, en la que las coordinadoras evaluaban los productos -estética y conceptualmente-, el nivel de desarrollo en el que se hallaban los emprendimientos y la trayectoria de los productores. En relación a la participación en estas ferias y al proceso de selección, una emprendedora asegura:

Ha salido en todos los periódicos de la zona...es como que le dieron un marco más...yo me sentía más contenida en ese marco de Cultura del municipio, viste, no era cualquier feria. Te daban ganas de participar y había gente que venía de capital... a participar, ¿eh? Había productos que eran de afuera...o sea, afuera de Quilmes, quiero decir. Y sí...eh...la selección del producto estaba buena porque veías que eran lindos productos, que no era como cualquier improvisado que venía a presentar las cosas. Entonces vos estando entre esos pares, te sentías mejor. O sea, que tu producto estaba más valorado.

En este mismo sentido, y sobre el proceso de selección, otras emprendedoras señalan:

Ellas se toman un tiempo para decidir y después te ponen en el listado de los que quedaron. Porque realmente los que quedaban eran buenos, ¿eh? Porque te digo que había mucho diseño bueno. Y cada edición tenías que presentar tus proyectos. No es que por haber quedado...

Conocí a gente y venía gente de otros lados. Eso estaba bueno también, que viniera gente de otro lado, que no sea solo de Quilmes. Venían de San Telmo, Palermo...Venía diseño y quedaba el bueno. Porque a veces que seas de Quilmes no significa que tu producto sea bueno también. 
De estos fragmentos se deduce que para las emprendedoras pasar por la instancia de selección -que se repetía en cada edición de la feria- no era una experiencia negativa o tediosa, sino que, por el contrario, era percibida como una forma de valorización de su labor como productoras y de los productos que manufacturaban. Por lo tanto, las emprendedoras preferían participar en una feria municipal a hacerlo en una feria privada, no sólo en términos económicos - por tratarse de un evento gratuito-, sino porque tenían que someterse a un proceso de selección y consideraban "prestigioso" el hecho de haber sido elegidas por sus propuestas (Vargas, 2013: 48).

Asimismo, se hace visible una jerarquización de las zonas urbanas, posicionando a CABA en un lugar privilegiado frente a las localidades de la zona sur del conurbano. Por lo tanto, el hecho de que productores de CABA expusieran en estas ferias de diseño de Quilmes, en especial los que solían hacerlo en barrios donde circulaban frecuentemente productos de "diseño", aportaba un valor mayor a estos eventos. Como sostiene Bourdieu, "el espacio físico es sólo el soporte vacío de las propiedades sociales de los agentes y las instituciones que, distribuyéndose allí, hacen de él un espacio social, socialmente jerarquizado" (Bourdieu, 2014b: 166). De esta manera, el valor social de los distintos barrios, según indica este autor, depende de la representación que los agentes se hacen del espacio social. Tanto para las coordinadoras como para las emprendedoras entrevistadas ciertos barrios localizados en CABA -como San Telmo y, en particular, Palermo- poseen una jerarquía mayor, al menos en lo que atañe a la producción y circulación de diseño, en comparación a otros espacios "periféricos", como por ejemplo Quilmes. Este reconocimiento del barrio de Palermo como un espacio dedicado al diseño (Miguel, 2009; Guerschman, 2010) es mencionado en diversas entrevistas: "Palermo es zona bien de diseño, pero también es carísimo estar ahí y a mí me queda lejos".

A su vez, el sitio elegido para realizar las ferias era, como indiqué, la Casa de la Cultura, localizada en el centro de Quilmes. La elección de este lugar no era casual, ya que el objetivo era, según las coordinadoras, que los emprendedores se mostraran "en un contexto lindo, un contexto armado", que pasaran por una selección y que luego el evento se llevara a cabo en un sitio "lindo", con una buena curaduría y acompañado de diversos espectáculos musicales. Al respecto, dos emprendedoras plantean: "la Casa de la Cultura, donde se hacían las ferias DAS, es un lugar precioso y gigante, muy bien ubicado". "Se generaba un ambiente de un producto puesto en Costa Salguero, en el mejor lugar”.

En relación a la distribución de los diversos estratos sociales en el espacio físico, cabe mencionar que la zona en la que se localizaba la feria es habitada, mayormente, por sectores medios y medios-altos. Esta información aportada por informantes clave es valiosa para comprender también cuál es el valor asignado al espacio, y las "centralidades periféricas" (Coulomb y Delgadillo, 2016). Las emprendedoras, asimismo, residen en el centro de Quilmes y en el centro de Bernal, y se adscriben bajo la categoría de "clase media". Esta categoría también es aplicable al análisis del público, ya que como afirman diversas emprendedoras: "Era gente con poder adquisitivo, gente joven, en su mayoría, que iba a buscar diseño". "El público era de clase media, gente que en general vivía ahí cerca".

Por ende, el público, al igual que las emprendedoras, residía, mayormente, en los barrios de clases medias - como el centro de Quilmes y de Bernal-, donde tenía lugar la feria, y a su vez pertenecían a esos sectores sociales. Además, eran jóvenes que estaban acostumbrados 
a consumir diseño, o que les interesaba acceder a este tipo de consumos, pero que en general adquirían esos productos en zonas de CABA, como Palermo, en donde su circulación ya estaba legitimada socialmente; los que aún no habían accedido a este tipo de bienes, los asociaban con esos lugares.

En conclusión, las coordinadoras del DAS, en su rol de intermediarias culturales, propiciaron un evento en donde, por un lado, daban a conocer las últimas tendencias en materia de diseño y, por el otro, concentraban "la demanda y oferta en un mismo lugar". De esta manera, vinculaban al público con los productores. Un público que, como afirmé anteriormente, era reacio a consumir productos de "diseño" en la zona sur, aunque asimismo proclives a este tipo de consumos. La estrategia de convocar a diseñadores que tuvieran sus locales en Palermo en las primeras ediciones de la feria significó, en este sentido, una forma de valorizar el proyecto del DAS y a los emprendimientos locales, y también ofrecer al público, como indicó una de las coordinadoras, "la posibilidad de ver productos de Palermo en Quilmes". Pero la propuesta de la feria implicaba también darles a los productores una oportunidad de exhibirse en un "contexto lindo", un lugar "bien ubicado", en donde pudieran "defender" sus productos y promover el concepto de sus marcas. Además, pasar por una instancia de selección y estar entre pares -también elegidos-, que en algunos casos provenían de otras ciudades, era experimentado por los emprendedores como un modo de valorizar su producción y su desempeño como productores.

\section{Vinculación, capacitación y concursos}

Paralelamente a las ferias de diseño, las coordinadoras del DAS pusieron en práctica otras estrategias de promoción y valorización de la producción quilmeña. Según indica una de ellas, las ferias eran:

La cara visible de DAS y es a donde todo el mundo, digamos, apuntaba a estar porque tenían un montón de cualidades: estaba buena la curaduría, la gente no pagaba...Pero nuestro eje estaba puesto en la vinculación, la capacitación, todo lo que tenía que ver con generar cuestiones relacionadas al diseño desde todo ámbito. Bueno, estaba acompañado también por una cuestión federal, que era todo lo que era el Mercado de Industrias Culturales...

En efecto, como se planteó al inicio de este artículo, a partir de mediados de la década del 2000 en adelante se constituyeron desde el Estado nacional diversas políticas públicas e instituciones dedicadas al desarrollo de las industrias culturales, con el propósito puntual de abarcar todas las zonas del país. La emergencia, entonces, del programa DAS está en íntima sintonía con estos esfuerzos por "federalizar" la producción de bienes culturales -y su acceso-. Así también, del fragmento citado, se deduce que uno de los ejes centrales del DAS fue la vinculación con otros espacios de circulación y producción de diseño. Al respecto, una de las coordinadoras expresa: 
Nosotros tratábamos primero de generar redes con organismos e instituciones del Estado o privadas que ya trabajaban con diseñadores. Si nosotros queríamos fortalecer la zona, teníamos que generar la situación, ponernos en contacto con otros lugares que estuvieran generando lo mismo.

"Generar la situación" -o, como en el caso de las ferias, "un contexto armado"- significaba crear algo que aún no existía o no al menos de forma organizada y nucleado bajo un único proyecto. La vinculación "con otros lugares que estuvieran generando lo mismo", y que gozaran de cierta legitimadad en el ámbito cultural, fue otro modo de crear el valor de los creadores y de sus creaciones y, asimismo, la creencia colectiva en dicho valor.

Esta articulación se dio con diversas instituciones. En primer lugar, las coordinadoras se pusieron en contacto con el MICA, organismo que aglutina, en encuentros anuales, la oferta nacional de industrias culturales, con el fin de generar intercambios y presentar la producción argentina a referentes del ámbito internacional. El DAS ha ocupado un stand, en las diversas ediciones del MICA, en el que expusieron varias emprendedoras, $y$ ha generado vínculos con otros productores y gestores culturales gracias a esa participación. Asimismo, el DAS y el Centro Metropolitano de Diseño (CMD) organizaron conjuntamente desayunos de negocios para diseñadores provenientes de distintas zonas del conurbano. Los datos provistos por el municipio indican que este encuentro posibilitó un "espacio de debate y construcción colectiva sobre el escenario del diseño en la zona sur" (informe de gestión, 2015).

De forma simultánea a las actividades de vinculación, las intermediarias culturales planificaron distintas capacitaciones gratuitas, destinadas a diseñadores y emprendedores, brindadas por reconocidos profesionales del ámbito de la moda y del diseño. Las capacitaciones consistían en talleres de producción de moda, moldería, sublimación, experimentación en fieltro, entre otros. Las coordinadoras señalan que organizaban las capacitaciones en función de las nuevas tendencias que aparecían en el ámbito del diseño, aunque sin descuidar las necesidades que surgían al dialogar con los emprendedores. Al relatar su experiencia en las capacitaciones, una emprendedora sostiene: "Hice el curso de sublimación de Araceli Pourcel, el de sublimación de telas. Ella es una diseñadora que es muy conocida".

Otra de las estrategias de "puesta en valor" de los productos y productores, impulsadas por el DAS, fue el armado del "catálogo de diseñadores quilmeños". Este material, de consulta y difusión de los proyectos productivos que integraron el programa, comenzó a editarse de forma anual, a partir de 2011, y se distribuía gratuitamente en formato impreso y virtual. Frases como "aquí exponemos la excelencia del diseño local" -que aparecen al inicio de los catálogos- evidencian una clara intención de parte de las intermediarias de valorizar los productos y darlos a conocer al público local. Al respecto, una emprendedora plantea que "a partir del catálogo, te invitaban a ferias más importantes...a partir de estar en un lugar, donde te podían encontrar, te ofrecían otras cosas".

Por último, en el marco del DAS, se organizaron desfiles y concursos de diseño de indumentaria, con distintas premisas, que contaron con la actuación de un jurado, compuesto por reconocidas diseñadoras y personalidades del mundo de la moda -como Andrea Saltzman, Ana Torrejón, Mariana Dapianno- encargado de evaluar las distintas prendas. 
Los ganadores del concurso - que contaba con tres primeros puestos- obtenían un reconocimiento simbólico, pero también monetario. A su vez, se organizó anualmente, como evento de cierre de fin de año, un desfile de "diseño de autor", en donde se mostraba "lo mejor de la moda de autor en nuestra ciudad" (informe de gestión, 2015). En relación a los concursos, una de las emprendedoras cuenta: "Había un jurado en el desfile organizado por el DAS. Yo saqué el tercer puesto y no lo podía creer...yo no conocía al jurado, porque recién empezaba, pero una amiga que estaba ya metida en el mundo del diseño, me dijo que eran re grosos".

Esto indica, volviendo a Bourdieu, que es en el campo mismo del diseño donde se engendran el valor de los productos y la creencia en ese valor (Bourdieu, 2014b). Para esta emprendedora que recién ingresaba al mundo del diseño, los jurados no despertaban en ella ningún sentimiento de admiración, pero para su amiga que ya conocía las reglas y dinámicas del campo -y sobre todo que creía en ellas- la opinión de éstos adquiría un valor especial. Por ende, lo que crea el poder del productor no es ni lo que fabrica ni tampoco la mera injerencia de los intermediarios culturales, sino que es el campo mismo, es decir, "el sistema de relaciones en conjunto" (Bourdieu, 1990a: 223).

\section{Reflexiones finales}

A lo largo de este artículo he examinado de qué manera se constituyó, a partir del año 2008, un circuito de diseño de indumentaria y accesorios en Quilmes, qué características adquirió y cuáles fueron los elementos simbólicos que contribuyeron a su consolidación. Este fenómeno pudo darse gracias a la confluencia de múltiples factores. Por un lado, la categoría "diseño" ya contaba con cierta notoriedad en la escena pública, desde comienzos de la década del 2000, en particular en CABA. Por otro lado, hacia fines de 2008, la industria textil y de indumentaria sufrió una desaceleración en su crecimiento, debido, a grandes rasgos, a las repercusiones que la crisis mundial tuvo en el país y al "conflicto del campo". Frente a esta situación, se implementaron distintas medidas anticíclicas, como el incremento de la regulación de las importaciones y un mayor estímulo del mercado interno, que favorecieron la emergencia de emprendimientos de indumentaria de pequeña escala dedicados a la producción de prendas de "diseño". Asimismo, hacia mediados de los años 2000, y en especial a partir de 2008, se inició un proceso de "jerarquización” de la cultura, que tuvo como objetivo "federalizar" la producción y circulación de bienes culturales. En este marco, se crearon diversas políticas públicas culturales -como el Programa DAS (Diseños al Sur) - y nuevas instituciones dedicadas al fomento de las industrias culturales, como el MICA, que tuvieron alcance nacional.

No obstante, como he demostrado, la planificación de políticas públicas no fue suficiente para generar una "atmósfera" propicia para la circulación de productos con el atributo del diseño (Miguel, 2009: 19). Para ello, se necesitó de la intervención de diferentes agentes, que colaboraron en la creación del valor de los productos y en la creencia en ese valor. En este sentido, las intermediarias culturales - coordinadoras del DAS- jugaron un rol central en la puesta en valor de los productos y productores, a través de la implementación de diversas estrategias -organización de ferias que requerían un proceso de selección y una 
curaduría; concursos y premios otorgados por consagrados actores del campo del diseño; capacitaciones brindadas por reconocidas diseñadoras, entre otras-. En conclusión, pudo conformarse un circuito de diseño en Quilmes y ser considerado por las emprendedoras y el público de la zona como un espacio "que aglutinaba el diseño", gracias a las políticas públicas y la disponibilidad de predios feriales para la comercialización de los productos (McRobbie, 2002), pero en especial debido a la participación de las intermediarias culturales, y diversos agentes del campo del diseño, en la producción de la "creencia colectiva" en el valor de los bienes y productores que integraron el circuito de diseño quilmeño.

\section{Notas}

1. Entiendo por circuito a un "circuito interpersonal", que se distingue por una frontera; un conjunto de lazos interpersonales; transacciones económicas; y formas específicas de intercambio (Zelizer, 2008).

2. En varios discursos, Cristina Fernández de Kirchner destacó "el auge" que la cultura alcanzó durante sus dos mandatos, entendiendo a la cultura desde su dimensión social e identitaria, pero a su vez como una forma de contribuir al desarrollo económico. A partir del 2003, el gobierno nacional invirtió "más de 24.000 millones de pesos en cultura", una inversión "como nunca se dio en Argentina". http://www.telam.com.ar/ notas/201505/105805-cristina-inauguracion-centro-cultural-nestor-kirchner.html (consultado el 08/10/2018).

3. Véase el decreto 641, por medio del que se creó el Ministerio de Cultura de Nación, en 2014, donde se menciona el objetivo de "jerarquizar" el área y garantizar su accionar en todas las jurisdicciones del país. https://www.lanacion.com.ar/1688374-por-decretocristina-kirchner-creo-el-ministerio-de-cultura (consultado 25/10/2018).

4. Según esta noción, el autor crea en soledad casi por inspiración divina, bastándose solamente de sus recursos imaginativos.

\section{Bibliografía}

Amar, A. y Tumini, L. (2012). “Comercio y empleo durante la crisis internacional: la dinámica de las exportadoras”, en: Macroeconomía, empleos e ingresos. Debates y políticas en Argentina frente a la crisis internacional 2008-2009, MTEySS-OIT, Buenos Aires, pp. 157-186.

Aronskind, R. (2012). "Modelos emergentes después de la crisis internacional. La reconfiguración del capitalismo mundial”, en: Macroeconomía, empleos e ingresos. Debates y políticas en Argentina frente a la crisis internacional 2008-2009, MTEySS- OIT, Buenos Aires, pp. 27-62.

Barthes, R. (1968). "La muerte del autor”, disponible en: http://www.cubaliteraria.cu/revista/ laletradelescriba/n51/articulo-4.html (consultado 29/10/2018).

Bourdieu, P. (1990a). "Alta costura y alta cultura”, en: Sociología y cultura, México, D.F., Editorial Grijalbo, pp. 215-224. 
(1990b). “YY quién creó a los creadores?”, en: Sociología y cultura, México, D.F., Editorial Grijalbo, pp. 225-238.

. (1995). Las reglas del arte. Génesis y estructura del campo literario, Barcelona, Editorial Anagrama.

Bourdieu, P. (2012). La distinción. Criterios y bases sociales del gusto, Madrid, Taurus. . (2014a). "El mercado de bienes simbólicos", en: El sentido social del gusto. Elementos para una sociología de la cultura, Buenos Aires, Siglo Veintiuno Editores, pp. 85-152. . (2014b). "La producción de la creencia. Contribución a una economía de los bienes simbólicos", en: El sentido social del gusto. Elementos para una sociología de la cultura, Buenos Aires, Siglo Veintiuno Editores, pp. 153-229.

Castells, M. y Schorr, M. (2015). "Cuando el crecimiento no es desarrollo. Algunos hechos estilizados de la dinámica industrial en la posconvertibilidad”, en: Cuadernos de Economía Crítica, Año 1, No 2, pp. 49-77.

CENDA (2010). La anatomía del nuevo patrón de crecimiento y la encrucijada actual. La economía argentina en el período 2002-2010, Cara o Ceca, Buenos Aires.

Colabella, L. (2014). "O te vamos a ir a buscar... un caso de brujería en los límites de la observación participante”, en: Guber, Rosana (comp.), Prácticas etnográficas. Ejercicios de reflexibilidad de antropólogas de campo, Buenos Aires, IDES-Miño y Dávila Editores, pp. 87-112.

Correa, M. E. (2018). Entre la industria y la autogestión, Buenos Aires, ExLibrisTeseoPress.

Coulomb, R. y Delgadillo, V. (coords.) (2016). Habitar la centralidad urbana, México, D. F., Universidad Autónoma Metropolitana.

Du Gay, P. et al. (1997), Doing Cultural Studies: the story of the Sony Walkman, London, Sage/The Open University.

Du Gay, P. (2003). Production of culture/Cultures of production, London, SAGE.

Guerschman, Bárbara (2010), "La marca comercial y el diseño: una reflexión antropológica sobre la producción, el consumo y el espacio”, Kula. Antropólogos del Atlántico Sur, Nro. 3, octubre, pp. 67-81.

Kulfas, M. (2017). Los tres kirchnerismos. Una historia de la economía argentina (2003-2015), Buenos Aires, Siglo Veintiuno Editores.

Mauss, M. (1979). Sociología y antropología, Madrid, Editorial Tecnos.

McRobbie, A. (2002). "Fashion culture: creative work, female individualization, Londres", Feminist Review, 71, pp. 52-62.

Miguel, P. (2009). "Los recorridos del diseño de indumentaria en la ciudad de Buenos Aires", Apuntes de investigación del CECYP, $\mathrm{n}^{\circ}$ 15, Buenos Aires, pp. 47-69.

. (2011). "Creatividad, economía y cultura en la ciudad de Buenos Aires 20012010”, en: Miguel, Paula y Lucas Rubinich (eds.), Creatividad, economía y cultura en la ciudad de Buenos Aires 2001-2010, Aurelia Rivera, pp. 45-68.

. (2013). Emprendedores del diseño. Aportes para una sociología de la moda, Buenos Aires, Eudeba.

Narotzky, S. (2016). "El proyecto en el modelo: Reciprocidad, capital social y la política del realismo etnográfico", (Con)textos, Nro. 6, febrero, pp. 84-99.

Negus, K. (2002). "The work of cultural intermediaries and the enduring distance between production and consumption”, Cultural Studies 16 (4), pp. 501-515. 
Vargas, P. (2013). Diseñadores y Emprendedores. Una etnografía sobre la producción y el consumo de diseño en Buenos Aires, La Plata, Al Margen.

Yúdice, G. (2008). El recurso de la cultura. Usos de la cultura en la era global, México, Gedisa. Zelizer, V. (2008). "Dinero, circuitos, relaciones íntimas", Revista Sociedad y economía, No. 14, junio, pp. 7-30.

\title{
Catálogos:
}

Catálogo de Diseñadores Quilmeños, DAS (2011, 2012, 2013).

MICA Produce Buenos Aires, 2015.

Informes:

Informe final de gestión del DAS, 2015.

\begin{abstract}
This article proposes to analyze the specificity of the symbolic goods, their relationship with the market and the construction of the collective belief in the value of the producers and the objects they manufacture, based on the contributions of Pierre Bourdieu to the sociology of culture. To address these issues I will analyze, punctually, the role of cultural intermediaries, within the framework of the various cultural public policies, in order to constitute and legitimize a clothing and accessories design circuit in the region of Quilmes, located in the south zone of Buenos Aires, between 2008-2015.
\end{abstract}

Keywords: symbolic goods - collective belief - cultural intermediaries - design - value circuit - public policies.

Resumo: Este artigo propõe analisar a especificidade dos bens simbólicos, sua relação com o mercado e a construção da crença coletiva no valor dos produtores e dos objetos que fabricam, com base nos aportes de Pierre Bourdieu à sociologia da cultura. Para abordar estas questões, vou analisar, pontualmente, o papel que exerceram os intermediários culturais, no marco das diversas políticas públicas culturais, para poder constituir e legitimar um circuito de desenho de vestuário e acessórios na região de Quilmes, localizada na zona sul de Buenos Aires, entre 2008-2015.

Palavras chave: bens simbólicos - crença coletiva - intermediários culturais - desenho valor - circuito - políticas públicas.

[Las traducciones de los abstracts fueron supervisadas por el autor de cada artículo] 